\title{
Organ Weight
}

National Cancer Institute

\section{Source}

National Cancer Institute. Organ Weight. NCI Thesaurus. Code C90429.

A measurement of the heaviness of a body organ. 